\title{
ANALYSIS OF THERMAL AND AQUEOUS SUSPENSION STABILITIES OF CHITOSAN BASED NANOENCAPSULATED VITAMINS
}

\author{
Douglas de Britto ${ }^{a, *}$, Flávia G. Pinola ${ }^{\mathrm{b}}$, Luiz H. C. Mattoso ${ }^{\mathrm{b}}$ and Odilio B. G. Assis ${ }^{\mathrm{b}}$ \\ aEmbrapa Semiárido, Rodovia BR-428, Km 152, 56302-970 Petrolina - PE, Brasil \\ ${ }^{b}$ Embrapa Instrumentação, R. XV de Novembro, 1.452, Centro, 13560-970 São Carlos - SP, Brasil
}

Recebido em 27/01/2016; aceito em 12/05/2016; publicado na web em 15/07/2016

\begin{abstract}
The food nutritional content is reduced as result of vitamins degradation. In order to minimize such losses, the encapsulation technique into polymeric nanoparticles (NPs) could offer an additional protection, extending the stability. The crosslinking formed by the ionic gelation process of chitosan and tripolyphosphate (Chi-TPP) has been widely used as an encapsulating matrix for several chemical compounds. In this way, Chi-TPP was used for encapsulation of C, B9 and B12 vitamins. The stability of the vitamins in NPs was evaluated in aqueous suspension by UV-Visible spectroscopy under different conditions: stored in dark, light exposure and effect of oxygen bubbling. The results indicated that encapsulation had a positive effect in preserving the vitamins, mainly vitamin $\mathrm{C}$. The encapsulation preserved $47 \%$ of the initial concentration of vitamin C by the tenth day and around $28 \%$ after 17 days. Conversely, in non-encapsulated controls (neutral and acidic medium) the losses were higher, reaching $13 \%$ by the tenth day and almost 3\% after 17 days of storage in both medium. When exposed to light and $\mathrm{O}_{2}$ the protection provided by the encapsulation was even greater. By thermogravimetric analysis, the pure and the encapsulated vitamins showed distinct thermal behavior confirming Chi-TPP as a potential encapsulation material.
\end{abstract}

Keywords: controlled release; vitamin degradation; nutrition; active food; thermogravimetric.

\section{INTRODUCTION}

Most of food nutrients and in special the vitamins are susceptible to fast degradation during processing, storage, and consumption. According to literature, ascorbic acid (vitamin C) is chemically stable in acid $\mathrm{pH}$, but unstable when in alkaline or neutral aqueous media. It also suffers intense action when subjected to oxygen, light and temperature and may reach values close to $100 \%$ of Maximum Cook Loose (MCL). In contrast, folic acid (vitamin B9) is quite stable in alkaline media, but unstable in neutral or acidic $\mathrm{pH}$ and likewise suffers degradation by oxygen, light and heat, also reaching a $100 \%$ MCL. Cyanocobalamin (vitamin B12) is relatively more stable and can withstand variations in $\mathrm{pH}$, although is susceptible to oxygen and light, presenting MCL near $10 \%$.

Van Bree et al., ${ }^{2}$ analyzing the degradation kinetics of ascorbic acid in fruit juice, reported that in non-stressed conditions the vitamin $\mathrm{C}$ degrades irreversibly according to a first order model. Even when conserved under optimal conditions of temperature and filling, most of natural juices lose 20 to $35 \%$ of their initial vitamin C content after one year storage. ${ }^{3}$ Attempts to fortify milk with vitamin $\mathrm{C}$ resulted in a loss of 35-40\% over 7 days when stored at $4{ }^{\circ} \mathrm{C}$ in the dark ${ }^{4}$ and when added to solid food as bread and cereals flakes, high rates of loss were observed (approximately 80 and 40\%, respectively). ${ }^{5}$ In the same way, folic acid also showed variation in stability during storage, mainly in enriched food. ${ }^{67}$ Exampling, powdered milk showed considerable losses of folic acid (60\% on average), after one year of storage. For the sterilized milk and ready-to-drink milk chocolate beverage, the losses of folic acid were 25 and $29 \%$, respectively, during the four months of storage. Concerning the vitamin B12, there is no data regarding its stability in active food, but questions has been aroused about the safe limit, degradation in the marketing process, efficacy and bioavailability of vitamin B12 fortified food. ${ }^{8}$

The addition of vitamins to animal food is also a concern. Coelho ${ }^{9}$

*e-mail: douglas.britto@embrapa.br reviewed the stress factors that can influence the stability of several added vitamins during processing and storage in premixes and feeds for ruminant diets, pointing to the effects of pelleting and extrusion over ingredients degradation. Guilherme et al., ${ }^{10}$ in an interesting work, evaluated the degradation of ascorbic acid when added to ration for fish against several factors as thermal stability, photochemistry and leaching, evidencing that vitamin content decreased approximately $60 \%$ after 30 days storage in an aqueous medium.

There are many ways of reducing the loss of bioactive and active ingredients in nutraceutical products, ${ }^{7,11-13}$ and, among them, the polymeric encapsulation has been used to protect susceptible vitamins against environmental or external impacts. ${ }^{12,13}$ This polymeric encapsulation can be achieved by micro- or nanoencapsulation techniques. ${ }^{13}$ The nanoencapsulation to obtain NPs generally is made by ionic gelation process that consists of setting an inter- and intramolecular crosslinking between a polyelectrolyte, e.g., chitosan (Chi) or trimethylchitosan (TMC), ${ }^{14}$ and a poly-ion as sodium tripolyphosphate (TPP). ${ }^{15,16}$ The driven force that maintain the stability of the system is the elelctrostatic interaction between ions of opposite charges. However, the characteristic of nanoparticle is highly dependent on the polymer and crosslinking agent concentrations, $\mathrm{pH}$ and presence of a third specie (specially, the encapsulated substance).

The feasibility of entrapping vitamins C, B9, and B12 into Chi/ TPP and TMC/TPP nanoparticles has previously been confirmed in our studies, in which structural and chemical changes were related and discussed by means of spectroscopic analyses (solid state ${ }^{13} \mathrm{C}$ NMR and Fourier Transformed Infrared). Atomic Force Microscopy and Scanning Electron Microscopy were also used to characterize nanoparticles shapes and size distribution. ${ }^{17}$ Release kinetics of vitamins C, B9, and B12 from Chi/TPP nanoparticles in aqueous medium were determined and related to specific vitamin properties as molecular weight and solubility. ${ }^{18}$ A significant observation from that study was to establish a quantitative relationship between the concentration of vitamins and nanoparticle structural characteristics such as size and zeta potential. 
In order to advance the understanding and the characterization of the protective effect generated by Chi/TPP nanoparticles in the vitamins encapsulation, the present work aims to analyze the vitamins stability (C, B9, and B12) in nanoencapsulated condition under different temperatures, oxidative atmospheres $\left(\mathrm{O}_{2}\right)$ and light conditions.

\section{EXPERIMENTAL SECTION}

\section{Materials}

Sodium tripolyphosphate (TPP) and medium molecular weight chitosan (Chi), 80\% deacetylated, were purchased from Aldrich Chemical Company Inc. (St. Louis, USA), and used as supplied. Other chemicals used were obtained from Synth (Rio de Janeiro, Brazil). Ascorbic acid (vitamin C), folic acid (vitamin B9) and cyanocobalamin (vitamin B12) were also supplied from Aldrich Chemical Company Inc. (St. Louis, USA).

\section{Synthesis of the nanoparticles and encapsulation of vitamins}

The nanoparticles were synthesized according to adopted methodology as previously described ${ }^{17}$ In summary, the process comprised a continuous addition of $50.0 \mathrm{~cm}^{3}$ of TPP solution at $0.7 ; 1.34$ and $1.38 \mathrm{mg} \mathrm{cm}^{-3}$, respectively for vitamins $\mathrm{B} 9, \mathrm{C}$ and $\mathrm{B} 12$, into $50.0 \mathrm{~cm}^{3}$ of $3.0 \mathrm{mg} \mathrm{cm}^{-3}$ of Chi solution. Chi was previously dissolved in an aqueous solution of acetic acid at $1 \%(\mathrm{v} / \mathrm{v})$. The system was magnetically stirred at room temperature during the addition of TPP drop wise solution to the $\mathrm{Chi} /$ vitamin mixture at a rate of $1.0 \mathrm{~cm} \mathrm{~min}^{-1}$. The stirring extended for more 15 minutes after the total addition. The amount of vitamins was kept at $15.0 \%$ (w/w), based on the chitosan weight. The final loaded particles have an average size of around $320 \mathrm{~nm}$. Physical-chemical characteristics of these nanoparticles are reported elsewhere. ${ }^{18}$

\section{Vitamin stability in aqueous suspension}

After encapsulation procedure, the degradation of vitamins was evaluated in aqueous suspension during storage time and under different conditions of oxidant medium $\left(\mathrm{O}_{2}\right)$ and light exposure. Evaluation of storage was performed by keeping samples of encapsulated vitamin $\mathrm{C}$ in an oven at a constant temperature of $30^{\circ} \mathrm{C}$, in absence of light, for three weeks. Twice a week, $1.0 \mathrm{~cm}^{3}$ aliquot was taken and stabilized with $1.0 \mathrm{~cm}^{3}$ of metaphosphoric acid solution at $6.0 \%$ (w/v). For encapsulated vitamins B9 and B12, the samples were stored at room temperature $\left(25 \pm 2{ }^{\circ} \mathrm{C}\right)$, in absence of light for three weeks. For these, aqueous solution of $0.1 \mathrm{M} \mathrm{KOH}+0.1 \mathrm{M} \mathrm{KH}_{2} \mathrm{PO}_{4}$ was used as a stabilizing agent.

Aqueous solutions of pure vitamins (non-encapsulated) were prepared and underwent the same procedures as the encapsulated ones to serve as a control for comparison. All vitamins (C, B9 and B12) were dissolved in deionized water in a ratio of $0.255 \mathrm{mg} \mathrm{cm}^{-3}$. Alkalinization of the solution with $0.1 \mathrm{M}$ of $\mathrm{NaOH}$ was necessary to allow vitamin B9 solubilization. For vitamin $\mathrm{C}$, an additional comparative control was prepared at $0.255 \mathrm{mg} \mathrm{cm}^{-3}$ in acetic acid solution at $1.0 \%(\mathrm{w} / \mathrm{v})$.

Because of its low stability, vitamin $\mathrm{C}$ was further investigated regarding the influence of light and oxidant medium $\left(\mathrm{O}_{2}\right)$. To assess the influence of the light, the suspension of nanoencapsulated vitamin $\mathrm{C}$ and its controls were exposed to natural and incandescent light $(\mathrm{Hg}$ vapor, $20 \mathrm{~W}$ ) on the laboratory bench along three weeks at room temperature.

The influence of oxygen was determined by bubbling $\mathrm{O}_{2}$ gas (Perez Oxigênio, 99.9\%) directly into the solutions (suspension of encapsulated vitamin $\mathrm{C}$ and its control) for 1 hour in alternative days with a moderate gas flux. The stability was expressed as the relative concentration with respect to amount initially solubilized. Curves and mathematical adjustment were performed using a Microcal Origin 9.0 software (OriginLab Co., Northampton, MA, USA).

\section{Entrapment capacity and thermal analysis}

The suspension of encapsulated vitamins was centrifuged at $14500 \mathrm{rpm}$ for 10 minutes (MiniSpin Plus, Eppendorf) before analyses. The amount of vitamins in each sample was determined using a UV-Visible spectroscopy model UV-1601PC (Shimadzu Corporation, Japan), in the spectral wavenumbers of $244,283,362 \mathrm{~nm}$ for vitamins C, B9 and B12, respectively. For the calculation, calibration curves were done for each vitamin from serial dilution of a stock vitamin solution at $0.225 \mathrm{mg} \mathrm{cm}^{-3}$. The solvents used to prepare the stock solutions were aqueous metaphosphoric acid at $3.0 \%(\mathrm{w} / \mathrm{v})$ for vitamin $\mathrm{C}$, and aqueous solution of $0.1 \mathrm{M} \mathrm{KOH}+0.1 \mathrm{M} \mathrm{KH}_{2} \mathrm{PO}_{4}$ for vitamins $\mathrm{B} 9$ and $\mathrm{B} 12$.

The thermogravimetric analysis was performed in a TGA-500 (TA Instruments, Brussels, Belgium), using $5.0 \mathrm{mg}$ of sample under nitrogen atmosphere (gas flow of $60 \mathrm{~cm}^{3} \mathrm{~min}^{-1}$ ). The samples were heated from room temperature to $500{ }^{\circ} \mathrm{C}$ at heating rate of $10^{\circ} \mathrm{C} \mathrm{min}{ }^{-1}$.

\section{RESULTS AND DISCUSSION}

\section{Stability of encapsulated vitamin in aqueous suspension}

After synthesis was completed, the suspension acquires opaque appearance with characteristic color of each vitamin. No precipitation of nanoparticles was observed during the experiment, indicating that Chi/TPP/vitamin forms a stable system without visual perception of particles agglomeration.

Analyses demonstrated that vitamins B9 and B 12 under both conditions are reasonably stable in aqueous media, not degrading extensively at room temperature $\left(\sim 25^{\circ} \mathrm{C}\right)$ over a period of 15 days storage (Figure 1). The variation in concentration can adequately be adjusted to a linear fit $(\mathrm{y}=a+b \mathrm{x})$, in which $b$ means the slope (inclination of the line), physically interpreted as the rate of concentration loss. For all adjusted data, the measured $b$ is very low, in the order of $10^{-4}$, indicating satisfactory stability with a tendency of minimal reduction in the concentration over time.

It is important to note that the measured concentrations for vitamin B12 are very similar in both conditions (diluted and encapsulated) indicating good interaction between polymer and this vitamin. Also depicted from the figure is that the amount of vitamin B9 into Chi-TPP matrix is slightly lower and is estimated in approximately $72 \%$ of the initial diluted concentration. The acid solvent used in chitosan gel formation (acetic acid) may have influenced the vitamin B9 stability, since it is known that this vitamin is sensitive to acidity. ${ }^{19}$

The vitamin $C$ behaves differently in aqueous media. The analyses indicate an inferior stability with a pronounced variation in the concentration over time as shown in Figure 2. The loss follows almost the same behavior either in neutral or acid medium in an evident exponential degradation. The adjusted curves for the vitamins provides an acceptable approximation to a non-linear regression fitting an exponential decay, with general equation:

$$
Y=y_{0}+A e^{-(x / t)}
$$

where $A$ is the interception with the ordination axis, reflecting the number of total molecules having sufficient energy to react, and $-(\mathrm{x} / \mathrm{t})$ express, in module, the rate of reaction, i.e., how fast the concentration curve drops with time. ${ }^{20}$ 


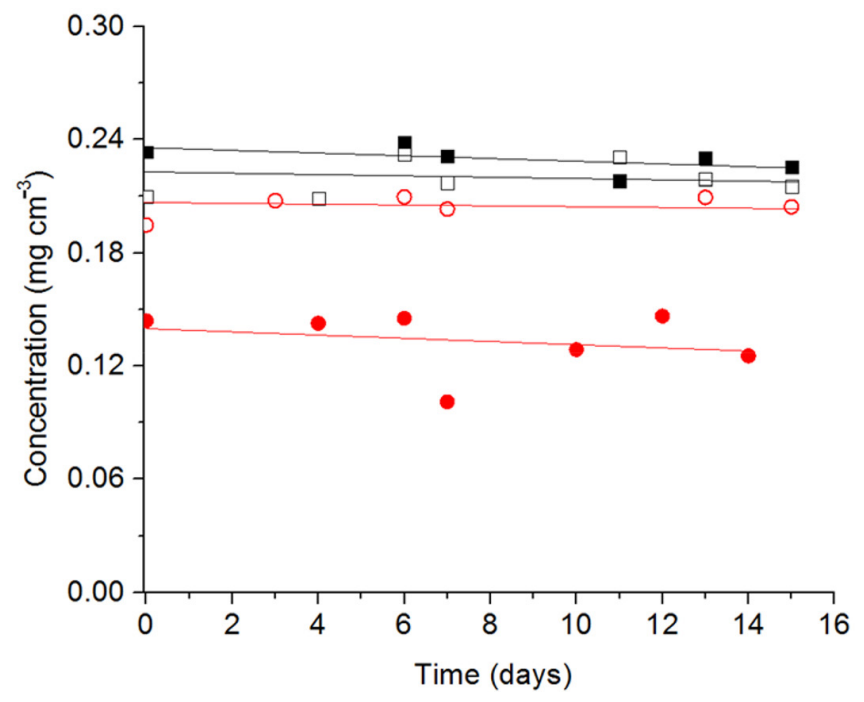

Figure 1. Variation of concentration of nanoencapsulated vitamins B9 (-) and $B 12(\mathbf{\square})$ in comparison with non-encapsulated vitamins $B 9(\bigcirc)$ and $B 12$ ( $\square$ ), during 15 days of storage in deionized water ( $p H$ 6.8) at room temperature and darkness

The encapsulated vitamin $\mathrm{C}$ also follows an exponential decay model, however, with less pronounced losses. By comparing with respect to initial value, the encapsulated condition maintains $47 \%$ of the initial concentration by the tenth day and around $28 \%$ after 17 days. These are high figures compared with non-encapsulated control (in neutral and acidic medium) in which the proportional concentration reaches $13 \%$ by the tenth day and drops to approximately $3 \%$ in the $17^{\text {th }}$ day. From the adjusted curves of Figure 2, the exponential power related to the speed of vitamin degradation along the 17 days storage can be determined as 0.066 for encapsulated samples and 0.334 for non-encapsulated vitamin, both in water. In a simple numerical comparison, these values indicate that vitamin C deteriorated $80 \%$ faster in diluted condition when compared to Chi/TPP encapsulation. When dissolved in $1 \%$ acetic acid solution the power is 0.230 , i.e., the degradation rate is approximately $70 \%$ faster than the encapsulated ones. This confirms that vitamin $\mathrm{C}$ has a different stability depending on its solubilizing medium. In this case,

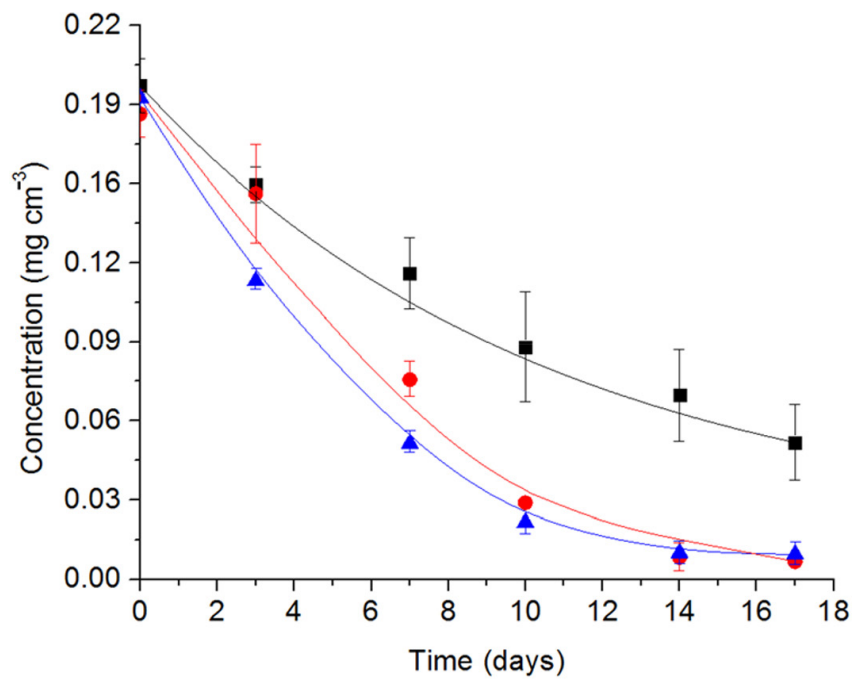

Figure 2. Variation of concentration of nanoencapsulated vitamins $C(\boldsymbol{\square})$ in comparison with non-encapsulated vitamins $C$ in deionized water $(\mathbf{O})$ and $1 \%$ acetic acid solution ( $\mathbf{\Delta})$ during 15 days at $30^{\circ} \mathrm{C}$ and darkness. The points were adjusted to exponential decay model (eq. 1), with $R^{2}$ values $\geq 0.90$ for all fits acetic acid is largely used for chitosan dissolution and has a negative effect on the vitamin $\mathrm{C}$ stability.

This model of degradation kinetic represents a pseudo-first-order irreversible approach (dependent only on one variable, in our case the time) and it is widely applied and cited in the literature..$^{21,2}$ Considering that initially all concentrations are the same $(\sim 0.19 \mathrm{mg}$ $\mathrm{cm}^{-3}$ ), it is evident by the curves parameters that the polymeric ChiTPP matrix provides protection, thereby reducing the degradation rates. Additionally, it is reported that the stability of vitamin $\mathrm{C}$ within biopolymeric nanoparticles is also better preserved when the system was subjected to different heat conditions. ${ }^{22}$

The loss of chemical agents initially retained inside polymeric matrix can involve two different mechanisms. According to Zhou et al. ${ }^{23}$ structural changes of the polymeric matrix may occur, simultaneously or not, to the diffusion of the agent to the medium. It is important to note that the vitamins achieved strong bonds with the polymeric matrix, as previous characterized, ${ }^{18}$ which reduces the diffusion to the medium before polymeric degradation starts. Accordingly, the use of chitosan derivative as encapsulation matrix (for example the $\mathrm{N}, \mathrm{N}, \mathrm{N}$-trimethylchitosan, which presents permanent charged groups) can promote improved ionic interactions between vitamins and nanoparticles making the retention more stable. ${ }^{24}$

Vitamin $\mathrm{C}$ was further investigated regarding $\mathrm{O}_{2}$ and light exposure (Figures 3 and 4). The degradation of vitamin $C$, when associated with light incidence, was found to follow the same exponential model, with kinetics parameters closer to those measured for time (Figure 2), and having a slightly better stability in acetic acid medium. Comparing to the initial concentration, the encapsulated samples by 15 days storage displayed a concentration equivalent of $37 \%$ of the initial amount, while the vitamin content was measured as $12 \%$ in acidic medium and as low as $7 \%$ in water.

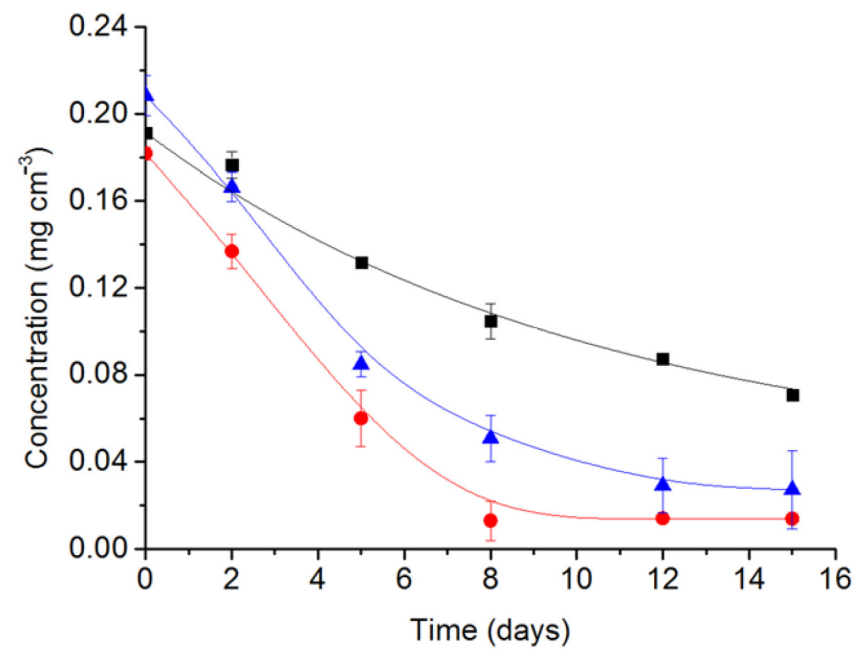

Figure 3. Variation of concentration of nanoencapsulated vitamins $C$ ( $\mathbf{\square}$ in aqueous suspension in comparison with non-encapsulated vitamins $C$ in deionized water (-) and $1 \%$ acetic acid $(\mathbf{\Delta})$ solutions, along storage time at room temperature, in constant presence of light. The points were adjusted to exponential decay model (eq. 1), with $R^{2}$ values $\geq 0.90$ for all fits

The protective benefits of encapsulation become more evident when examining the effect of oxygen on degradation as depicted by the curves in Figure 4. The use of Chi-TPP as encapsulation matrix proved to be very effective as a barrier to $\mathrm{O}_{2}$ allowing, after 20 days, the maintenance of values close to $50 \%$ with respect to initial vitamin amount. For non-encapsulated vitamin, the loss at this time reaches $80 \%$, with rapid degradation in the first week, approaching to zero after 10 days. 
The degradation of vitamin $\mathrm{C}$ is a complex catabolic mechanism that involves a number of oxidative and reductive reactions, breaking down the molecule into smaller units. The formation of intermediates products is determined by several factors, mainly the aerobic or anaerobic pathway. According to model proposed by Yuan and Chen, ${ }^{25}$ under aerobic conditions the ascorbic acid is easily oxidized, transforming in dehydroascorbic acid and further hydrolyzed to furfural (2-furoic acid, 3-hydroxy-2-pyrone), whereas, under anaerobic conditions, it degrades directly to furfural. Additionally, humidity and water presence have to be taken into account since vitamin $\mathrm{C}$ is very sensible to water effect (Figure 2) and studies have shown that the rates of degradation increase with increasing water activity $\left(\mathrm{w}_{\mathrm{a}}\right){ }^{26}$ That is a concern for processing vitamin $\mathrm{C}$ enriched products which involve liquid and intermediate moisture foods. According to Laing et al. ${ }^{27} \mathrm{~W}_{\mathrm{a}}$ is 0.69 in the presence of $20 \%$ of water (w/w) and reaches 0.90 when the water is $32 \%$ in mass. When encapsulated into chitosan, the vitamin $\mathrm{C}$ half-life is around 31 days when $\mathrm{w}_{\mathrm{a}}$ is 0.31 and falls to 4.3 days as $\mathrm{w}_{\mathrm{a}}$ is increased to $0.74 .^{12}$ Vitamin leaching into water is also an important consideration when dealing with animal food, especially fish food, which may result in losses of up to $71 \%$ of the initial vitamin $\mathrm{C}$ added by diffusion to the water prior to fish consumption. ${ }^{10}$ In such cases, polymeric encapsulation can be an important technology in promoting a better retention and vitamin stability.

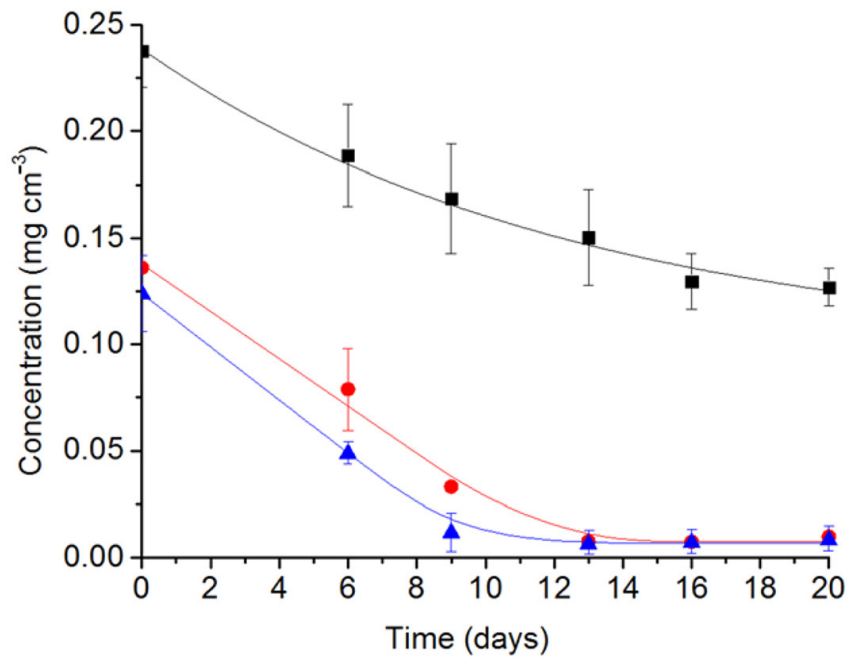

Figure 4. Variation of concentration of nanoencapsulated vitamins $C(\boldsymbol{\square})$ in comparison with non-encapsulated vitamins $C$ in deionized water (O) and $1 \%$ acetic acid ( $\mathbf{\Delta})$ solutions with bubbling of $\mathrm{O}_{2}$ gas at room temperature. The points were adjusted to exponential decay model (eq. 1), with $R^{2}$ values $\geq 0.90$ for all fits

\section{Thermogravimetric analysis}

Although the degradation mechanism in aqueous phase is completely different than in solid state, the thermogravimetric analysis of nanoencapsulated vitamins is important in providing additional information about vitamin stability when complexed with the polymeric matrix. The evolution of decomposition under temperature differs for each tested samples as shown in Figure 5. Additionally, the degree of mass loss also differs for the same vitamin in encapsulated and non-encapsulated conditions. It is observed from DTG thermograms (Figure 6) that both pure vitamins B12 and B9 showed mass loss with events starting near 40 and $92{ }^{\circ} \mathrm{C}$ respectively, corresponding to the desorption of physical and chemical bounded water. Hence, no dehydration can be identified for pure vitamin $\mathrm{C}$ in agreement to thermal analysis as presented in the literature. ${ }^{28-31}$ According to Jingyan et al.,$^{32}$ for ascorbic acid decomposition, the main thermal event simultaneously yields $\mathrm{H}_{2} \mathrm{O}, \mathrm{CO}_{2}, \mathrm{CO}, \mathrm{HCOOH}$ and $\mathrm{CH}_{4}$, forming furfural which will be degraded in the following step. In addition, it should be noted that all the vitamins have multiple stages of thermal degradation after water loss.

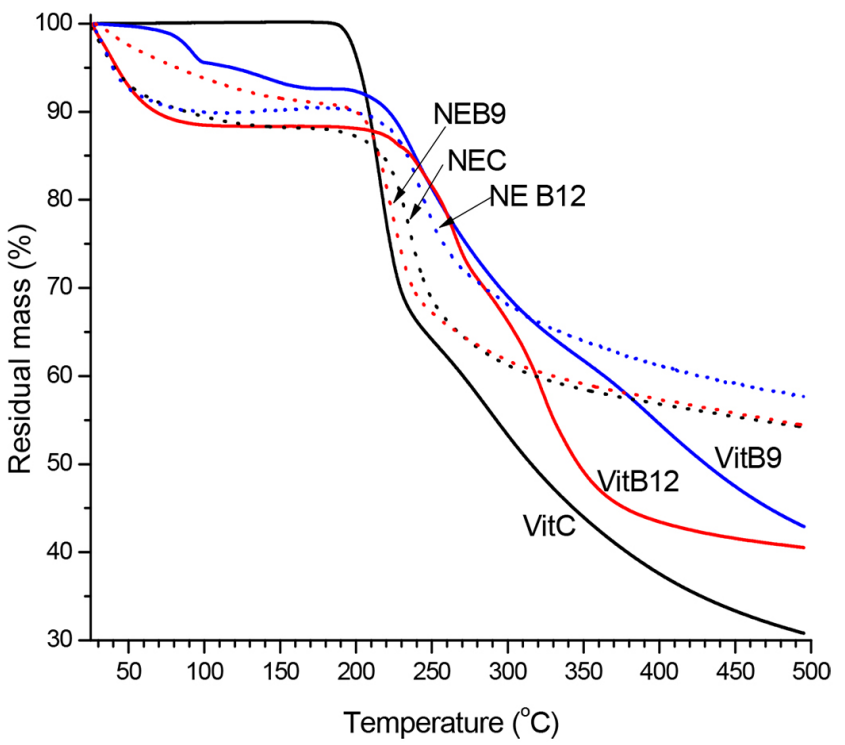

Figure 5. Thermogravimetric curve for pure vitamins $C$ (-), B9 (-) and B12 (-) and nanoencapsulated vitamins $N E C$ (...), NEB9 (...) and NEB12 (...) at $\mathrm{N}_{2}$ atmosphere and heating rate of $10^{\circ} \mathrm{C} \mathrm{\textrm {min } ^ { - 1 }}$

For vitamin B9, the thermal degradation occurs by the cleavage of the folate molecules due to the loosing of glutamic acid moieties. In this process, the folates (a mixture of polyglutamyl tetrahydrofolates and glutamate residues) are split between the C-9 and N-10 bond, yielding a pteridine and p-aminobenzoylglutamate compounds which have no biological activity. ${ }^{33}$ For B12, the thermal loss of mass is associated to the removal of cyanide group and ammonia, both acting as a strong stabilizer of vitamin structure. Chemical details of these vitamins structures were presented and discussed in a previous publication. ${ }^{17}$ For encapsulated samples, despite the evidence of water desorption (around $40-50{ }^{\circ} \mathrm{C}$ ) mainly due to water bonded to the polymer, no additional events (characteristics to vitamin degradations) were observed (Figure 6).

The absence of the peaks can be explained by the low amount of vitamin retained in comparison to the polymeric nanoparticles mass and a strong crosslinking between vitamin, Chi and TPP. As a result, only one event is recorded corresponding to degradation undergone by the entire system. Any analytical comparisons by TG analysis are unfeasible, owing to the stability of vitamin in encapsulated and non-encapsulated conditions. However, it is worth observing that there is an appreciable difference in peaks obtained by DTG for each encapsulated sample: nanoencapsulated vitamin C, B9 and B12 have maximum in the DTG curve at 236,243 and $222^{\circ} \mathrm{C}$, respectively (Figure 6 and Table 1). For pure nanoparticle made of chitosan and TPP (without any encapsulated molecule) the literature presents a DTG degradation peak at $294{ }^{\circ} \mathrm{C},{ }^{34}$ being indicative of the effective presence and interaction between vitamins into nanoparticles. The identified peaks and main thermal results are summarized in Table 1.

\section{CONCLUSION}

The results indicate that the nanoencapsulation with chitosan and TPP offers good stabilization for vitamin $\mathrm{C}$ in aqueous solution, even 
Table 1. Thermal parameters from TGA curves

\begin{tabular}{|c|c|c|c|c|c|c|}
\hline \multirow{2}{*}{ Vitamin } & \multirow{2}{*}{$\begin{array}{l}\text { Dehydration } \\
\text { range }\left({ }^{\circ} \mathrm{C}\right)\end{array}$} & \multicolumn{4}{|c|}{ Thermal events: Maximum decomposition temperature $\left({ }^{\circ} \mathrm{C}\right)$} & \multirow{2}{*}{$\begin{array}{l}\text { Residual mass } \\
\text { at } 500{ }^{\circ} \mathrm{C}(\%)\end{array}$} \\
\hline & & $\mathrm{Td}_{1}$ & $\mathrm{Td}_{2}$ & $\mathrm{Td}_{3}$ & $\mathrm{Td}_{4}$ & \\
\hline B9 & $40-$ & 239 & 396 & - & - & 43 \\
\hline B12 & $92-$ & 226 & 244 & 265 & 323 & 41 \\
\hline $\mathrm{C}$ & $\mathrm{NO}^{* *}$ & 215 & 290 & - & - & 31 \\
\hline NEB9* & 135 & 243 & - & - & - & 56 \\
\hline NEB12 & 100 & 222 & - & - & - & 54 \\
\hline NEC & 85 & 236 & - & - & - & 54 \\
\hline
\end{tabular}

$* \mathrm{NE}=$ nanoencapsulated $* * \mathrm{NO}=$ not observed.

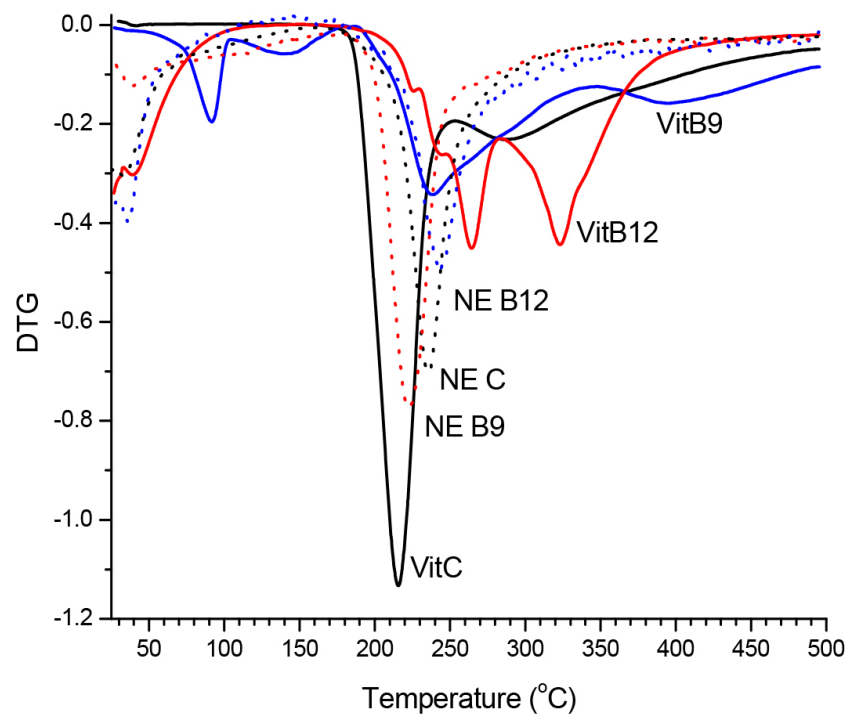

Figure 6. Respective DTG curves of the thermogravimetric curves depicted in Figure 5

in the presence of light and $\mathrm{O}_{2}$. In the best of the conditions, based on the final concentrations, the gain in amount for nanoencapsulated vitamin $\mathrm{C}$ was near $30 \%$ high than the non-encapsulated one. This highlight the significance and effectiveness of nanoencapsulation to preserve instable molecules. Vitamins B9 and B12 appear to have a better stability under the conditions analyzed, in which encapsulation had little or no influence on their stability in aqueous medium. The results attained for vitamin $\mathrm{C}$ reinforce the potential of Chi/TPP as a material for vitamin encapsulation suitable for applications in food, cosmetic and pharmacological areas. Additionally, by thermal degradation analysis, it was demonstrated that the presence of vitamin decreases overall system stability, by weakening the bonds established between the polymers which form the nanoparticles.

\section{ACKNOWLEDGMENT}

The authors are grateful to FINEP/MCT, Embrapa (Rede AgroNano), FAPESP, FACEPE, CNPq and CAPES for the financial support.

\section{REFERENCES}

1. Gregory III, J. F. In Chemical Changes in Food during Processing; Richardson, T., Finley J. W., eds.; Springer: New York, 1985, cap. 17.

2. van Bree, I.; Baetens, J. M.; Samapundo, S.; Devlieghere, F.; Laleman, R.; Vandekinderen, I.; Noseda, B.; Xhaferi, R.; de Baets, B.; de Meulenaer, B.; Food Chem. 2012, 134, 207.
3. de Ritter, E.; Food Technol. 1976, 30, 43.

4. Rosenthal, I.; Rosen, B.; Bernstein, S.; Milchwissenschaft 1993, 48, 676.

5. Steele, C.; Cereal Foods World 1976, 21, 538.

6. Lima, J. A.; Catharino, R. R.; Godoy, H. T.; Ciênc. Tecnol. Aliment. 2004, 24, 82 .

7. Masiá, R. P.; Nicolás, R. L.; Periago, M. J.; Ros, G.; Lagaron, J. M. Rubio, A. L.; Food Chem. 2015, 168, 124.

8. Dary, O.; Food Nutr. Bull. 2008, 29, S214.

9. Coelho, M.; Proceedings of the 13th Annual Florida Ruminant Nutrition Symposium, Gainesville, FL, USA. 2002.

10. Guilherme, R. F.; Cavalheiro, J. M. O.; Brasileiro, O. L.; Prado, J. P. S.; Cavalheiro, T. B.; Cienc. Agrotecnol. 2009, 33, 1153.

11. Sekhon, B. S.; Nanotechnol., Sci. Appl. 2010, 3, 1.

12. Pulido, A.; Beristain, C. I.; Rev. Mex. Ing. Quim. 2010, 9, 189.

13. Alishahi, A.; Mirvaghefi, A.; Tehrani, M. R.; Farahmand, H.; Shojaosadati, S. A.; Dorkoosh, F. A.; Elsabee, M. Z.; Food Chem. 2011, 126, 935.

14. Campana-Filho, S. P.; de Britto, D.; Quim. Nova 2009, 32, 1461.

15. Gan, Q.; Wang, T.; Cochrane, C.; McCarron, P.; Colloids Surf., B 2005 , $44,65$.

16. Chen, F.; Zhang, Z.-R.; Huang, Y.; Int. J. Pharm. 2007, 336, 166.

17. de Britto, D.; Moura, M. R.; Aouada, F. A.; Mattoso, L. H. C.; Assis, O. B. G.; Food Hydrocolloids 2012, 27, 487.

18. de Britto, D.; Moura, M. R.; Aouada, F. A.; Pinola, F. G.; Lundstedt, L. M.; Assis, O. B. G.; Mattoso, L. H. C.; Macromol. Res. 2014, 22, 1261.

19. Biamonte, A. R.; Schneller, G. H.; J. Am. Pharm. Assoc. 1951, 40, 313.

20. van Boekel, M. A. J. S.; Compr. Rev. Food Sci. Food Saf. 2008, 7, 144.

21. Sakai,Y.; Watanabe, H.; Takai, R.; Hasegawa, T.; J. Food Process. Preserv. 1987, 11, 197.

22. Jang, K.-I.; Lee, H. G.; J. Agric. Food Chem. 2008, 56, 1936.

23. Zhou, S.; Deng, X.; Li, X.; J. Controlled Release 2001, 75, 27.

24. de Britto, D.; Frederico, F. R.; Assis, O. B. G. Polym. Int. 2011, 60, 910.

25. Yuan, J. P.; Chen, F.; J. Agric. Food Chem. 1998, 46, 5078.

26. Bell, L. N.; Labuza, T. P.; Moisture sorption: Influence, Practical aspects of isotherm measurement and use, $2^{\text {nd }}$ ed., AACC Press: St. Paul, 2000.

27. Laing, B. M.; Schulueter, L.; Labuza, T. P.; J. Food Sci. 1978, 43, 1440.

28. Oteroa, R. L. S.; Galvão, R. K. H.; Araújo, M. C. U.; Cavalheiro, E. T. G.; Thermochim. Acta 2011, 526, 200.

29. Juhász, M.; Kitahara, Y.; Takahashi, S.; Fuji, T.; J. Pharm. Biomed. Anal. 2012, 59, 190.

30. Goldstein, S.; Duca, A.; Thermochim. Acta 1982, 59, 211.

31. Vora, A.; Riga, A.; Dollimore, D.; Alexander, K.; J. Therm. Anal. Calorim. 2004, 75, 709 .

32. Jingyan, S.; Yuwen, L.; Zhiyong, W.; Cunxin, W.; J. Pharm. Biomed. Anal. 2013, 77, 116.

33. Blakley, R. In The biochemistry of folic acid and related pteridines; Newbergen, H., Taton, E. L., eds.; North Holland: Amsterdam, 1969. Vol. 13.

34. Wang, M.; She, Y.; Xiao, Z.; Hu, J.; Zhou, R.; Zhang, J.; Carbohydr. Polym. 2014, 101, 812. 